\title{
A Retrospective Study of 75 Cases of Splenic Abscess
}

\author{
H. Sreekar • Vivek Saraf • Ashok C. Pangi • \\ H. Sreeharsha $\cdot$ Ravi Reddy $\cdot$ Gautam Kamat
}

Received: 19 December 2010 / Accepted: 26 October 2011 /Published online: 9 November 2011

(C) Association of Surgeons of India 2011

\begin{abstract}
Splenic abscess is an uncommon and lifethreatening condition. Due to its nonspecific clinical picture, it remains a diagnostic challenge. Multiple radiological modalities are used for the diagnosis. In this retrospective study we analyzed 75 patients treated between 1999 and 2009. The patients were divided into three groups depending on the treatment received. Group I $(n=14)$ consisted of patients treated with only antibiotics, Group II $(n=19)$ patients were treated with percutaneous drainage and Group III $(n=42)$ with splenectomy. We tried to establish epidemiologic and clinical features and therapeutic options in splenic abscess. Our study suggests
\end{abstract}

\footnotetext{
H. Sreekar $(\bowtie)$

Department of Plastic Surgery, Christian Medical College,

Vellore 632004, India

e-mail: drsreekarh@yahoo.com

V. Saraf

Department of gastroenterology,

Amrita Institute of Medical Sciences and Research Centre,

Cochin, Kerala, India

A. C. Pangi

Department of General Surgery,

KLE's PK Hospital and Research Centre,

Belgaum, Karnataka, India

H. Sreeharsha

SMS Medical College,

Jaipur, India

R. Reddy

Vijaynagar Institute of Medical Sciences,

Bellary, Karnataka, India

G. Kamat

Goa Medical College,

Goa, India

that percutaneous drainage is a safe and effective alternative to surgery especially in unilocular or bilocular abscesses thus allowing preservation of the spleen. It should be considered as the first line of treatment although splenectomy remains the final definitive procedure if percutaneous drainage fails.

Keywords Splenic abscess · Percutaneous drainage . Splenectomy

\section{Introduction}

A splenic abscess is an uncommon but life-threatening condition with an incidence of occurrence ranging from $0.1 \%$ to $0.7 \%$ in various series [1-3]. Splenic abscesses occur in diverse clinical scenarios and is a common result of splenic trauma, haematogenous or contiguous spread of bacteria. Patients with splenic abscesses have concomitant risk factors such as diabetes mellitus or immunocompromised conditions like AIDS [4, 5]. The diagnosis is usually delayed because of vague symptoms such as abdominal pain, fever and vomiting. Splenic abscesses are associated with increased mortality and complication rates. However, with the advent and easy access to improved imaging, this condition can be diagnosed early with better accuracy. Although selected patients can be treated solely with antibiotics, splenectomy has generally been the definitive surgical intervention in the treatment of splenic abscesses. With the evolution of better radiological techniques like ultrasound and CT scans, percutaneous drainage under guidance has added a new dimension to the management of splenic abscesses [6,7]. The purpose of this study is to review our experience and analyse the efficacy of these treatment modalities of splenic abscesses, in our setting. 


\section{Materials and Methods}

This study was conducted in a tertiary referral centre. The medical records of all the cases of splenic abscesses admitted between 1999 and 2009 were reviewed. Data regarding patient demographics and clinical characteristics such as age, sex, symptoms, signs, radiologic features, predisposing conditions, treatment, bacteriologic profile, complications and outcomes were recorded. The main clinical features like fever, abdominal pain, nausea and vomiting and tender splenomegaly were noted. Predisposing factors such as diabetes mellitus, tuberculosis, HIV/AIDS, concomitant liver disease, malignancies and immunocompromised states were also tabulated.

On clinical suspicion of having developed splenic abscesses, patients were subjected to haematologic and radiologic investigations such as ultrasound and $\mathrm{CT}$ scan of the abdomen. The leucocyte counts, erythrocyte sedimentation rate (ESR) and results of the radiologic investigations regarding solitary or multiple abscesses and any other incidental findings were recorded. The patients were then subjected to different treatment modalities like percutaneous/ open drainage, splenectomy or were given only antibiotics. The bacteriologic profile following the intervention was tabulated. The duration of the hospital stay, complications and eventual outcomes were noted and the data compared between the groups receiving different treatments.

The patients were divided into three groups depending on the treatment received. Group I $(n=14)$ consisted of patients treated with only antibiotics, Group II $(n=19)$ consisted of patients who were treated with percutaneous drainage and Group III $(n=42)$ consisted of patients who were treated with splenectomy.

All data were statistically analysed with Chi-square test and one-way ANOVA. A $P$ value $<0.05$ was considered significant.

\section{Observations and Results}

A total of 75 patients were included from the study period. The mean age of these patients was 34.98 years (range: between 3 and 88 years). The male-female ratio was 52:23 (Table 1). The mean duration of symptoms at the time of presentation was 3.1 days. The common presenting symptoms were fever $(89 \%)$, abdominal pain (84\%), nausea and vomiting (48\%). Of these patients, $33 \%$ also had a clinically palpable spleen. Leucocytosis $(\mathrm{WBC}>11.000)$ was observed in $67 \%$ and raised ESR ( $>25 \mathrm{~mm} / \mathrm{h}$ ) in $73 \%$ of these patients. The most common predisposing factor was diabetes mellitus and was present in 20 patients $(27 \%)$. Other factors were HIV/AIDS (16\%), pulmonary tuberculosis $(12 \%)$, parenchymal liver disease $(8 \%)$, malignancy
$(7 \%)$ and trauma (7\%). Of these patients, $10(14 \%)$ had more than one predisposing factor. There was no statistically significant difference among Groups I, II and III in terms of demographic characteristics, presenting clinical features and predisposing factors.

Thirty-four (45\%) patients were diagnosed by undergoing ultrasonography; 28 (37\%) by CT scan of the abdomen, and in $13(17 \%)$ patients both the modalities were used (Table 2). Multiple radiological investigations were carried out more in patients planned for intervention. Thirty-nine (52\%) patients had a single abscess cavity, and 34 (45\%) patients had multiple cavities. The common incidental findings were parenchymal liver disease (18\%), pleural effusion $(7 \%)$, abdominal lymphadenopathy (4\%), calculus cholecystitis $(3 \%)$, pancreatitis $(3 \%)$ and renal cysts (3\%) (Fig. 1).

Of the 39 cases diagnosed with single splenic abscess, 19 were treated with percutaneous drainage. Percutaneous drainage was not attempted in any of the patients with multiple abscesses. Of the 14 patients, 4 were treated solely with antibiotics, and 16 of the 42 patients were treated with splenectomy had a solitary abscess. Bacteriological examination (blood and/or pus culture) was conducted in 65 patients. Of these, $46 \%$ of the cases were monomicrobial, while $43 \%$ did not show any growth on culture and $11 \%$ were polymicrobial in nature (Table 3). The most common organisms grown were Esherichia coli (20\%) and Staphylococcus aureus (20\%) followed by Klebsiella pneumonia $(11 \%)$.

In the group treated only with antibiotics, the average duration of hospital stay was 13.71 days (SD 9.08) with a survival rate of $85.71 \%$. Two patients in this group succumbed to sepsis. The patients of Groups II and III had an average hospital stay of 11.42 and 15.58 days, respectively. The survival rates among Groups II and III were $94.73 \%$ and $95.24 \%$, respectively (Table 4 ). Of the two patients in the splenectomy group who expired, one succumbed to continuing sepsis and the other to postoperative pneumonia.

Complications related to the respiratory system (39.7\%) were most common followed by sepsis $(10.95 \%)$, wound infection $(4.10 \%)$, paralytic ileus $(2.73 \%)$, deep vein thrombosis $(4.10 \%)$ and meningitis $(1.36 \%)$. Five $(6.84 \%)$ patients had multiple complications. Of the 73 patients, 46 (63.01\%) had at least one complication (Table 5). Of the 29 patients who had lung complications, $23(79.31 \%)$ belonged to Group III. Half the cases (4) of septicaemia was from Group I and two cases each from the other two groups.

\section{Discussion}

A splenic abscess, though uncommon, has been increasingly diagnosed. The reasons are increased number of immuno- 
Table 1 Characteristics of the 75 patients of splenic abscess

\begin{tabular}{lccc}
\hline Characteristic & Antibiotics only & Percutaneous drainage & Splenectomy \\
\hline Age (years) & 34.1 years & 39.6 years & 33.2 years \\
Sex: M/F & $14(8: 6)$ & $19(15: 4)$ & $42(29: 13)$ \\
Duration of symptoms (Days) & 3.4 & 2.9 & 3.1 \\
Symptoms and signs (No. of patients) & & & \\
Abdominal pain & $9(64 \%)$ & $16(84 \%)$ & $36(86 \%)$ \\
Fever & $12(86 \%)$ & $15(79 \%)$ & $38(91 \%)$ \\
Nausea and vomiting & $6(43 \%)$ & $8(42 \%)$ & $21(50 \%)$ \\
Palpability of spleen & $3(21 \%)$ & $4(21 \%)$ & $17(41 \%)$ \\
Investigations & & $14(74 \%)$ & $25(60 \%)$ \\
Leukocytosis $\left(>11,000 / \mathrm{mm}^{3}\right)$ & $10(71 \%)$ & $14(74 \%)$ & $27(64 \%)$ \\
Raised ESR (>25 mm/ h) & $12(86 \%)$ & & \\
Predisposing factors & & $6(32 \%)$ & $8(26 \%)$ \\
Diabetes mellitus & $3(21 \%)$ & $3(16 \%)$ & $3(7 \%)$ \\
HIV/AIDS & $1(7 \%)$ & $1(6 \%)$ & $5(12 \%)$ \\
Liver disease & $2(14 \%)$ & $2(11 \%)$ & $2(5 \%)$ \\
Tuberculosis & $2(14 \%)$ & - & $1(2 \%)$ \\
Malignancy/ Chemotherapy & - & $2(11 \%)$ & $3(7 \%)$ \\
Trauma & - & & \\
Others & $4(29)$ & & \\
\hline
\end{tabular}

compromised patients and better imaging techniques [8]. Spleen has a vital role in the immunologic function; hence splenectomised patients have a higher incidence of infections and post-operative morbidity. Though splenic abscesses are currently managed primarily by percutaneous drainage, splenectomy still has a major role to play $[9,10]$.

In this study, the mean age of the patients was 34.98 years and was considerably lower than that reported in other studies $[1,11]$. This might be attributed to better access to radiological techniques and health care in the region. There was no significant difference in terms of the demographic data between the three treatment groups. The cause of splenic abscesses is often due to haematogenous spread or spread from contagious organs [9]. Increasing prevalence of diabetes mellitus, HIV/AIDS and liver disease, due to changing lifestyle trends, predispose the patients to splenic abscesses. In this study, 12 of the 73 patients were HIV-infected. In all these patients, multiple splenic abscesses were detected. Though three of these patients expired, the prognostic role of HIV cannot be

Table 2 Diagnostic radiology

\begin{tabular}{lccc}
\hline & $\begin{array}{l}\text { Antibiotics } \\
\text { only (14) }\end{array}$ & $\begin{array}{l}\text { Percutaneous } \\
\text { drainage (19) }\end{array}$ & $\begin{array}{l}\text { Splenectomy } \\
(42)\end{array}$ \\
\hline $\begin{array}{l}\text { Ultrasound } \\
\quad \text { Single/ Multiple) }\end{array}$ & $9(3 / 6)$ & $7(7 / 0)$ & $18(6 / 12)$ \\
CT scan (Single/ Multiple) & $4(1 / 3)$ & $8(8 / 0)$ & $16(6 / 10)$ \\
Both (Single/ Multiple) & $1(0 / 1)$ & $4(4 / 0)$ & $8(4 / 4)$ \\
\hline
\end{tabular}

ascertained. These patients generally present with the clinical triad of fever, abdominal pain, nausea and vomiting along with a palpable spleen on examination. Blood examination reveals raised ESR and leucocytosis, though these are not reliable in immuno-suppressed patients. The clinical findings and blood examination results were similar in all the three groups.

Bacteria are usually the pathogenic agents that account for splenic abscesses [12]. Rarely, fungi and protozoa are encountered, especially in the immuno-suppressed patients. Both gram-negative and gram-positive organisms have been implicated in splenic abscesses [11, 13, 14]. S. aureus and $E$. coli $(20 \%$ each) were the most common bacteria

\section{Incidental radiological Findings}

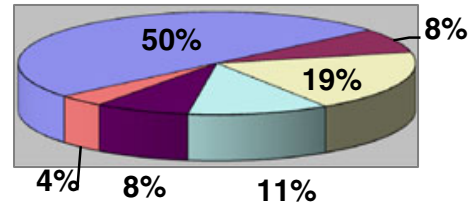

$\square$ Pleural effusion

$\square$ Abdominal lymphadenopathy

$\square$ Renal cyst

口Ovarian cyst

Fig. 1 Incidental radiological findings 
Table 3 Bacteriologic profile

\begin{tabular}{lc}
\hline Organism isolated & Number of patients \\
\hline Escherichia coli & $13(20 \%)$ \\
Streptococcus pyogenes & $2(3 \%)$ \\
Streptococcus pneumonia & $1(2 \%)$ \\
Klebsiella pneumonia & $7(11 \%)$ \\
Bacteroides fragilis & $2(3 \%)$ \\
Pseudomonas aeruginosa & $2(3 \%)$ \\
Staphylococcus aureus & $13(20 \%)$ \\
Proteus mirabilis & $1(2 \%)$ \\
Bacillus cereus & $1(2 \%)$ \\
Fungus & - \\
Monomicrobial & $30(46 \%)$ \\
Polymicrobial & $7(11 \%)$ \\
No growth & $28(43 \%)$ \\
Culture not sent & 10 \\
\hline
\end{tabular}

isolated from the patients in this study, followed by $K$ pneumoniae (11\%). In about $11 \%$ of the patients, multiple organisms were isolated, while none were isolated in $43 \%$.

Ultrasound and CT scan of the abdomen were the major radiological modalities used in the diagnosis. The radiologic and diagnostic study of preference for splenic abscesses is an abdominal examination by CT scans [15]. CT scans can characterise the contents of the abscess cavity and also reveal the uni/multilocularity of the abscesses. CT scans can also reliably differentiate splenic abscesses from splenic cysts and haematomas. Additionally, the location of abscess, relation of spleen to other visceral structures is delineated, thereby helping in planning for a percutaneous drainage. Ultrasound has a comparable accuracy in the detection of splenic abscesses. In this study, 28 patients underwent CT scans, 34 underwent ultrasound examination, and 13 patients underwent both. There was no significant difference between the two modalities in terms of detection of splenic abscesses and the uni/multilocularity nature of the same. In the radiological test, parenchymal liver disease (18\%) followed by pleural effusion, abdominal lymphadenopathy and pancreatitis were the most common incidental findings observed. Themanagement per se of the splenic abscess condition was not directly affected by these findings.

Table 4 Comparisons of the three groups

\begin{tabular}{lccl}
\hline Group & $\mathrm{n}$ & $\begin{array}{l}\text { Duration of hospital } \\
\text { stay (days) }\end{array}$ & Survival rate \\
\hline Antibiotics alone & 14 & $13.71 \pm 9.08$ & $12 / 14(85.71 \%)$ \\
Percutaneous drainage & 19 & $11.42 \pm 7.78$ & $18 / 19(94.73 \%)$ \\
Splenectomy & 42 & $15.58 \pm 5.75$ & $40 / 42(95.24 \%)$ \\
\hline
\end{tabular}

Table 5 Complications in the three groups

\begin{tabular}{lcccc}
\hline Complication & $\begin{array}{l}\text { Antibiotics } \\
\text { alone }\end{array}$ & $\begin{array}{l}\text { Percutaneous } \\
\text { drainage }\end{array}$ & Splenectomy & $\begin{array}{l}\text { Combined } \\
(\%)\end{array}$ \\
\hline $\begin{array}{l}\text { Lung infection } \\
\text { Septicaemia }\end{array}$ & 1 & 5 & 23 & $29(39.72)$ \\
$\begin{array}{l}\text { Wound } \\
\text { infection }\end{array}$ & 4 & 2 & 2 & $8(10.95)$ \\
$\begin{array}{l}\text { Paralytic ileus } \\
\begin{array}{l}\text { Deep vein } \\
\text { thrombosis }\end{array}\end{array}$ & 0 & 0 & 3 & $3(4.10)$ \\
$\begin{array}{l}\text { Meningitis } \\
\text { Multiple }\end{array}$ & 0 & 0 & 2 & $2(2.73)$ \\
$\quad$ complications & 0 & 1 & 2 & $3(4.10)$ \\
Total (Patients) & 5 & 0 & 1 & $1(1.36)$ \\
\hline
\end{tabular}

Fourteen patients were treated with antibiotics alone, 19 with percutaneous drainage and 42 with splenectomy. Percutaneous drainage is generally the preferred treatment modality in unilocular or bilocular abscesses and also when the content appears subjectively thin enough to be drained. Surgical treatment is preferred for more than two abscesses $[14,16,17]$. The location of the abscess is also important, with percutaneous drainage being preferred for peripherally situated abscesses. It is also preferred in patients who are critically ill or are unfit for general anaesthesia. Simple needle aspiration for peripheral splenic abscesses has also been attempted in some cases. The other advantages of drainage procedures are the absence of abdominal spillage, lesser costs, better acceptance and avoidance of the operative risks. Relative contraindications to percutaneous drainage include multiple or septated abscesses, anatomically inaccessible abscesses, coagulopathies, ascites or associated diseases requiring surgical procedures [18]. The drainage is generally done under ultrasound or CT guidance using 8-French to 20 -French catheters. The introduction of these catheters is usually associated with the spontaneous drainage of the pus. Poor drainage condition requires radiological verification of the position of the tube. In this study, multiple catheters were not used. Complications associated with percutaneous drainage of splenic abscesses include haemorrhage, septicemia and injury to other abdominal organs, empyema, pneumothorax and fistula. In this study, the complications associated with percutaneous drainage were lung infection (5/19), septicemia (2/19) and deep vein thrombosis (1/19). There was no case of adjacent organ injury or pneumothorax during the insertion.

Splenectomy has been the most effective and definitive procedure for managing splenic abscesses. Most studies report mortality rates of $0 \%$ to $20 \%$ during open splenectomy for splenic abscesses [11, 14, 19]. Although higher morbidity rates are associated with this procedure, it remains the only viable treatment option, especially for 
patients with multiple abscesses, failed percutaneous drainage, and also for recurrent abscesses. Of the 42 patients who underwent splenectomy, the mortality rate was $4.76 \%$ and complications were observed in $78.57 \%$ of them. The common complications were lung infection (23/42), wound infection (3/42), septicemia (2/42), paralytic ileus (2/42) and deep vein thrombosis $(2 / 42)$. Multiple complications were noted in $11.9 \%$ of these patients. The duration of hospital stay was 15.58 days, which was considerably longer as compared to 13.71 days in the antibiotic group and 11.42 days in the percutaneous drainage group, respectively.

\section{Conclusions}

A splenic abscess is an uncommon but fatal entity. Early diagnosis requires a high index of suspicion and prompt radiological investigation. This study suggests that percutaneous drainage is a safe and effective alternative to surgery, especially in unilocular or bilocular abscesses, allowing preservation of the spleen. It should be considered the first line of treatment, although splenectomy remains the final definitive procedure if percutaneous drainage fails.

\section{References}

1. Chun CH, Raff MJ, Contreras L, Varghese R, Waterman N, Daffner R et al (1980) Splenic abscess. Medicine (Baltimore) 59 (1):50-65

2. Lawhorne TW Jr, Zuidema GD (1976) Splenic abscess. Surgery 79:686-689

3. Gadacz TR (1985) Splenic abscess. World J Surg 9:410-415
4. Nelken N, Ignatius J, Skinner M, Christensen N (1987) Changing clinical spectrum of splenic abscess. A multicenter study and review of the literature. Am J Surg 154:27-34

5. Simson JN (1980) Solitary abscess of the spleen. Br J Surg 67:106-110

6. Thanos L, Dailiana T, Papaioannou G et al (2002) Percutaneous CT-guided drainage of splenic abscess. AJR 179:629-632

7. Gleich S, Wolin DA, Herbsman H, Rockaway F (1988) A review of percutaneous drainage in splenic abscess. Surg Gyn Ob 167:211-216

8. Farres H, Felsher J, Banbury M, Brody F (2004) Management of splenic abscess in a critically ill patient. Sorg Laparosc Endosc Percutan Tech 14:49-52

9. Zerem E, Bergsland J (2006) Ultrasound guided percutaneous treatment for splenic abscesses: the significance in treatment of critically ill patients. World J Gastroenterol 12(45):7341-7345

10. Choudhury SR, Rajiv C, Pitamber S, Akshay S, Dharmendra S (2006) Management of splenic abscess in children by percutaneous drainage. J Pediatr Surg 41:e53-e56

11. Nelken N, Ignatius J, Skinner M, Christensen N (1987) Changing clinical spectrum of splenic abscess. A multicenter study and review of the literature. Am J Surg 154:27-34

12. De Bree E, Tsiftsis D, Christodoulakis M, Harocopos G, Schoretsanitis G, Melissas J (1998) Splenic abscess: a diagnostic and therapeutic challenge. Acta Chir Belg 98:199-202

13. Chang KC, Chuah SK, Changchien CS, Tsai TL, Lu SN, Chiu YC et al (2006) Clinical characteristics and prognostic factors of splenic abscess: a review of 67 cases in a single medical center of Taiwan World. J Gastroenterol 12(3):460-464

14. Green BT (2001) Splenic abscess: report of six cases and review of the literature. Am Surg 67:80-85

15. Faught WE, Gilbertson JJ, Nelson EW (1989) Splenic abscess: presentation, treatment options, and results. Am J Surg 158:612-614

16. Chou YH, Hsu CC, Tiu CM, Chang T (1992) Splenic abscess: sonographic diagnosis and percutaneous drainage or aspiration. Gastrointest Radiol 17:262-266

17. Hadas-Halpren I, Hiller N, Dolberg M (1992) Percutaneous drainage of splenic abscesses: an effective and safe procedure. Br J Radiol 65:968-970

18. Gerzof SG, Johnson WC, Robbins AH et al (1985) Expanded criteria for percutaneous abscess drainage. Arch Surg 120:227-232

19. Phillips GS, Radosevich MD, Lipsett PA (1997) Splenic abscess: another look at an old disease. Arch Surg 132:1331-1335 